\title{
OPTIMAL DESIGN OF AN IP/MPLS OVER DWDM NETWORK
}

\author{
Eduardo Canale $^{1}$, Claudio Risso ${ }^{2 *}$ and Franco Robledo ${ }^{2}$
}

Received February 28, 2013 / Accepted October 21, 2013

\begin{abstract}
Different approaches for deploying resilient optical networks of low cost constitute a traditional group of NP-Hard problems that have been widely studied. Most of them are based on the construction of low cost networks that fulfill connectivity constraints. However, recent trends to virtualize optical networks over the legacy fiber infrastructure, modified the nature of network design problems and turned inappropriate many of these models and algorithms. In this paper we study a design problem arising from the deployment of an IP/MPLS network over an existing DWDM infrastructure. Besides cost and resiliency, this problem integrates traffic and capacity constraints. We present: an integer programming formulation for the problem, theoretical results, and describe how several metaheuristics were applied in order to find good quality solutions, for a real application case of a telecommunications company.
\end{abstract}

Keywords: telecommunications network, multi-layer network design, metaheuristics.

\section{INTRODUCTION}

In this paper, we address the problem of finding the optimal - minimum cost - configuration of a logical topology over a fixed optical network. The input data-set is constituted by the optical layer topology - DWDM network -, the client nodes of the data network - IP/MPLS nodes and the potential links between them, as well as the data traffic demands to satisfy between each pair of nodes and the per-distance-cost in the optical network associated with the bitrates of the optical connections to deploy over it. The decision variables are: what data links do we have to implement, which bitrate must be assigned to each of them and what path do their optical implementations have to follow in the optical layer. For being a feasible solution a configuration must be capable of routing every traffic demand over the remaining active links of the data layer, for every single physical link failure.

Because of the changes in the technology this model is significantly different from former models - referred in next section -, so are the algorithms to find solutions.

\footnotetext{
*Corresponding author

${ }^{1}$ Institute of Mathematics and Statistics, University of the Republic, Montevideo, Uruguay.

2 Institute of Computer Science, University of the Republic, Montevideo, Uruguay.

E-mails: \{canale, crisso, frobledo\}@ fing.edu.uy
} 
We proved this problem is NP-Hard (Theorem 4.1) and due to its complexity we developed several metaheuristics to find good quality solutions for real size scenarios. Best results were found with GRASP. To achieve good performance, many theoretical results needed to be integrated during the design process of the algorithms.

A remarkable feature of this work is that we analyzed the performance of the proposed metaheuristics using real-world scenarios provided by the Uruguayan national telecommunications company (ANTEL).

The main contributions of this article are:

i) a model to represent a common network overlay design problem;

ii) theoretical results useful to find and discard solutions under certain hypotheses;

iii) the design of a GRASP metaheuristic suitable to find good quality solutions;

iv) the experimental evaluation based on real-world network scenarios.

This paper is organized as follows. A history perspective is given in Section 2. A mixed-integer programming model will be presented in Section 3. In Section 4 we analyze the intrinsic complexity of the problem and some theoretical results useful to construct exact solutions for simple but important cases. In Section 5 we describe a real-world application case with many scenarios, present a battery of metaheuristics used to find good quality solutions, benchmark and analyze their results. Finally, in Section 6 we analyze the most important results of this work.

\section{HISTORIC PERSPECTIVE}

Some decades ago the increasing importance of the telephony service pushed most telecommunications companies (TELCOs) to deploy optical fiber networks. In order to guarantee appropriate service availability, these networks were designed in such a way that several independent paths were available between each pair of nodes, and in order to optimize these large capital investments several models and algorithms were developed. Already the optimal design of a single layer network is a challenging task that has been considered by many research groups, see for instance $[6,9,18]$. Throughout this work this optical network is referred to as the physical layer.

Some years afterwards, the exponential growth of Internet traffic volume, demanded for higher capacity networks. This demand led to the deployment of dense wavelength division multiplexing (DWDM) technology. This technology allows multiplexing several connections - lightpath connections - over one single cable of optical fiber using different wavelengths, and rapidly became very popular with telecommunications companies because it allowed them to expand the capacity of their networks without laying more fiber. Today, DWDM has turned out to be the dominant network technology in high-capacity optical backbone networks. Repeaters and amplifiers must be placed at regular intervals for compensating the loss in optical power while the signal travels along the fiber; hence the cost of a ligthpath is proportional to its length over 
the physical layer. DWDM supports a set of standard high-capacity interfaces (e.g. 1, 2.5, 10 or $40 \mathrm{Gbps}$ ). The cost of a connection also depends of the capacity but not proportionally. For economies of scale reasons, the higher the bitrate the lower the per-bandwidth-cost. The client nodes together with these lightpath connections form a so-called logical layer on top of the physical one.

The increasing number of per-physical-link connections - intrinsic to DWDM - may cause multiple logical link failures from a single physical link failure (e.g., fiber cut). This issue led to the development of new multi-layer models aware of the stack of network layers. Most of these models share in common the $1+1$ protection mechanism, i.e., for every demand two physically independent logical paths must be provided, such that in case of any single physical link or even node - failure, at least one of them survives. The following references $[7,10]$ are good examples of this kind of models. These multi-layer models are suitable for certain families of logical layer technologies such as synchronous optical networking (SONET) or synchronous digital hierarchy $(\mathrm{SDH})$ since both standards have $1+1$ protection as their native protection mechanism. As in these models, in our case, we assume that the physical layer is already installed and cannot be changed.

During many years the connections of IP networks were implemented over SONET/SDH - for simplicity we will only mention SDH from now on -. As a consequence, the IP layer - the third and top layer of this stack - rarely suffered unplanned topology changes. Most recently, multiprotocol label switching (MPLS), traffic engineering extensions for dynamic routing protocols (e.g. OSPF-TE, ISIS-TE), fast reroute algorithms (FRR) and other new features were added to the traditional IP routers. This new technology bundle known as IP/MPLS, opens a competitive alternative against traditional protection mechanisms based on SDH.

Since IP/MPLS allows recovering from a failure in about 50ms, capital savings may come from the elimination of the intermediate SDH layer. Another improvement of this technology is that the number of paths to route demands between nodes is not pre-bounded; so it might exist in fact a different feasible configuration for most failure scenarios. Moreover, there is no need to preestablish all of these paths explicitly. If the appropriate information is fed to the routing protocols and the network is designed with care, the dynamic routing algorithms usually construct solutions of very good quality. Since IP/MPLS allows the elimination of an intermediate layer, manages Internet traffic natively, and makes possible a much easier and cheaper operation for virtual private network (VPN) services, it is gaining relative importance every day.

Setting aside technical details, the IP/MPLS technology does not fit well with two natural features of the SDH technology. The first one is the need of SDH to keep different demands between the same nodes. In IP/MPLS networks all the traffic from one node to another follows the same path in the network referred to as IP/MPLS tunnel. Although possible, splitting the traffic between a pair of nodes into more than one tunnel requires complex configurations. The second remarkable difference is how these technologies handle the existence of parallel links in the logical layer. In SDH the existence of parallel links is typical but in IP/MPLS parallel links may conflict with some applications so we will avoid them. 
We refer to this problem as MORNDP (Multi-Overlay Resilient Network Design Problem). Solving the MORNDP implies:

i) design the data network, by defining the links to be include in the logical layer and the capacity assigned to each one;

ii) determine the lightpath for each link in the data network; these paths are fixed, so every physical link failure propagates to all logical links that use it;

iii) provide the data network tolerance to single failures on the transport network, by determining paths for the IP/MPLS tunnels, one per-each physical failure, so when a fault arises, the network can route all traffic demands without violating the capacities of the logical links;

iv) minimize the cost of the designed data network.

\section{MATHEMATICAL MODEL}

We will now introduce the basic mixed-integer programming model that arises from the detailed interaction of technologies.

Parameters. The physical network is represented by an undirected graph $(V, P)$, and the logical network is represented by another undirected graph $(V, L)$. Both layers share the same set of nodes. The links of the logical layer are potential - admissible logical links - while the links of the physical layer are definite. In both graphs the edges are simple since multigraphs are not allowed in this model.

For every different pair of nodes $p, q \in V$ is known the traffic volume $d_{p q}$ to fulfill along the unique path (tunnel) this traffic follows throughout a logical layer configuration. These paths are unique at every moment, but in case of link failures they may change to follow an alternate route. For simplicity we assume that the traffic volume is symmetric (i.e. $d_{p q}=d_{q p}$ ).

Let $\hat{B}=\left\{b_{1}, \ldots, b_{\bar{B}}\right\}$ be the set of possible bitrate capacities for the lightpaths on the physical layer and therefore for the links of the logical one. Every capacity $b \in \hat{B}$ has a known perdistance cost $c_{b}$. For economies of scale reasons it holds that if

$$
b^{\prime}<b^{\prime \prime} \text { then }\left(c_{b^{\prime}} / b^{\prime}\right)>\left(c_{b^{\prime \prime}} / b^{\prime \prime}\right) .
$$

Since both graphs of this model are simple and undirected, we will express links as pairs of nodes. For every physical link $(i j)$ is known its length $l_{i j}$.

Variables. This model comprises three classes of variables. The first class is composed of the logical link capacity variables. We will use boolean variables $\tau_{p q}^{b}$ to indicate whether or not the logical link $(p q) \in L$ has been assigned with the capacity $b \in \hat{B}$. As a consequence the capacity of the logical link $(p q)$ could be computed as $\sum_{b \in \hat{B}} b \cdot \tau_{p q}^{b}$.

The second class of variables determines how are going to be routed the logical links over the physical network. If $\sum_{b \in \hat{B}} \tau_{p q}^{b}=1$ then the logical link $(p q) \in L$ was assigned with a capacity, 
it is going to be used in the logical network and requires a lightpath in the physical one. $y_{p q}^{i j}$ is a boolean variable that indicates whether or not the physical link $(i j) \in P$ is being used to implement the lightpath of $(p q)$.

Since lightpaths cannot automatically recover from a link failure, whenever a physical link $(i j)$ fails all the logical links $(p q)$ such that $y_{p q}^{i j}=1$ do fail as well. The only protection available in this model is that of the logical layer. For demands being protected against single physical link failures, is necessary to have a feasible route through the remaining active logical links.

The third and final class of variables is that that determines how the IP/MPLS tunnels are going to be routed against any particular failure in a physical link. ${ }^{r s} x_{p q}^{i j}$ is a boolean variable that indicates whether the logical link $(p q) \in L$ is going to be used or not, to route traffic demand $d_{r s}>0$, under a faulty condition in the physical link $(i j) \in P$.

NOTE: To keep the nomenclature of the variables as easy as possible we always placed: logical links subindexes at bottom right position, physical links subindexes at top right position and demands subindexes at top left position.

Constraints. This problem comprises three groups of constraints. The first group of constraints establishes the rules that the routes of the lightpaths must follow to be feasible.

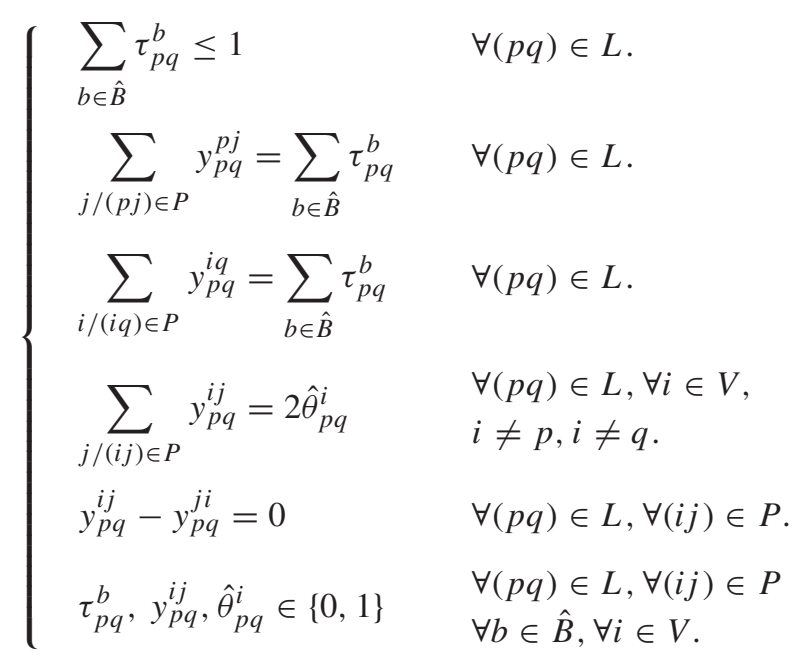

The meaning of constraints in group equation (1) is the following: (i) establishes that the number of capacities assigned to every logical link is at most $1-$ it could be 0 if the link is not going to be used -.

The constraints (ii) and (iii) guarantee that if any particular link $(p q) \in L$ was assigned with a capacity $\left(\sum_{b \in \hat{B}} \tau_{p q}^{b}=1\right)$ then there must exist one and only one outgoing - or incoming physical link used for its lightpath.

Before going any further we will introduce a set of auxiliary variables $\hat{\theta}_{p q}^{i}$. These variables are defined for every combination of logical links $(p q) \in L$ and physical nodes $i \in V$. Hence, (iv) guarantees flow balance for routing the lightpaths through the remaining - not terminal - nodes. 
Finally $(v)$ guarantees that the lightpaths go back and forth through the same path, while $(v i)$ stands the integrity of the variables.

The second group of constraints establishes the rules that the routes of the IP/MPLS tunnels must follow in the logical layer.

The meaning of the constraints in equation (2) is similar to those of equation (1) except for $(i)$. The inequalities in $(i)$ were added to guarantee that whatever the faulty scenario is $((i j) \in P)$, its associated routing configuration over the logical network keeps the aggregated traffic load below the link capacity for every data link $(p q) \in L$.

Constraints (ii) and (iii) from equation (1) and equation (2) are equivalent, except for the fact that in the latter the existence of a tunnel relies on the existence of demand and this is known in advance.

Another remarkable point is that equation (2) has as many possible routing scenarios as arcs in $P$, so the number of variables is much greater than those of equation (1).

Variables ${ }^{r s} \hat{\mu}_{p}^{i j}$ are homologous to $\hat{\theta}_{p q}^{i}$; so are constraints from (iv) to (vi).

$$
\begin{cases}\sum_{r s: d_{r s}>0} d_{r s} \cdot{ }^{r s} x_{p q}^{i j} \leq \sum_{b \in \hat{B}} b \cdot \tau_{p q}^{b} & \forall(p q) \in L, \forall(i j) \in P \\ \sum_{q /(r q) \in L}{ }^{r s} x_{r q}^{i j}=1 & \forall d_{r s}>0, \forall(i j) \in P \\ \sum_{p /(p s) \in L}{ }^{r s} x_{p s}^{i j}=1 & \forall d_{r s}>0, \forall(i j) \in P \\ \sum_{q /(p q) \in L}{ }^{r s} x_{p q}^{i j}=2 \cdot{ }^{r s} \hat{\mu}_{p}^{i j} & \forall d_{r s}>0, \forall(i j) \in P \\ { }_{r s} x_{p q}^{i j}-{ }^{r s} x_{q p}^{i j}=0 & \forall p \in V, p \neq r, p \neq s \\ { }_{r s} x_{p q}^{i j},{ }^{r s} \hat{\mu}_{p}^{i j} \in\{0,1\} & \forall d_{r s}>0, \forall(p q) \in L \\ & \forall(i j) \in P \\ & \forall d_{r s}>0, \forall(p q) \in L \\ & \forall(i j) \in P, \forall p \in V\end{cases}
$$

Before proceeding any further we must notice that equation (1) and equation (2) are not independent. Many logical links may not be available for routing after a physical link failure. Which logical links are in this condition, relies on how the lightpaths were routed in the physical layer. Specifically, if some logical link $(p q)$ uses a physical link $(i j)$ for its lightpath implementation then this logical link cannot be used to route any tunnel under $(i j)$ failure scenario.

$$
{ }^{r s} x_{p q}^{i j} \leq 1-y_{p q}^{i j} \quad \forall r s: d_{r s}>0, \forall(p q) \in L, \forall(i j) \in P .
$$

The group of constraints equation (3) prevents from using $(p q)$ to route any traffic $\left({ }^{r s} x_{p q}^{i j}=\right.$ $\left.0, \forall r s: d_{r s}>0\right)$ in any failure scenario which affects this link (when $y_{p q}^{i j}=1$ ). 
Objective. The function to minimize is the sum of the cost of every logical link. According on what capacity was assigned to a logical link there is an associated per-distance-cost $\left(c_{b}\right)$, and according on how the corresponding lightpath was routed over the physical layer it has an associated length $\left(\sum_{(i j) \in P} l_{i j} y_{p q}^{i j}\right)$.

The product of both terms is the cost of a particular logical link and the sum of these products for all the logical links is the total cost of the solution. The direct arithmetic expression for the previous statement would be

$$
\sum_{(p q) \in L}\left(\sum_{b \in \hat{B}} c_{b} \tau_{p q}^{b}\right)\left(\sum_{(i j) \in P} l_{i j} y_{p q}^{i j}\right)=\sum_{(p q) \in L,(i j) \in P, b \in \hat{B}} c_{b} l_{i j} \cdot \tau_{p q}^{b} y_{p q}^{i j}
$$

Although straightforward, this approximation is inappropriate because is non-linear.

The subproblem equation (4) expresses the objective value with an equivalent linear expression. We used the real variable ${ }^{b} \eta_{p q}^{i j}$ instead of $\tau_{p q}^{b} y_{p q}^{i j}$ and added some extra constraints to guarantee the consistency.

The consistency comes from the following observations. The result of $\tau_{p q}^{b} y_{p q}^{i j}$ is also a boolean variable, and since ${ }^{b} \eta_{p q}^{i j}$ is being multiplied by a positive constant in a minimization problem it will take its lowest value whenever this is possible. This value would be zero because of constraints (iii) of equation (4).

$$
\begin{cases}\min \sum_{\substack{(p q) \in L \\(i j) \in P \\ b \in \hat{B}}} c_{b} l_{i j} \cdot{ }^{b} \eta_{p q}^{i j} & \\ { }^{b} \eta_{p q}^{i j} \geq \tau_{p q}^{b}+y_{p q}^{i j}-1 & \forall(p q) \in L, \forall(i j) \in P, \\ & \forall b \in \hat{B} . \\ { }^{b} \eta_{p q}^{i j} \geq 0 & \forall(p q) \in L, \forall(i j) \in P, \\ & \forall b \in \hat{B} .\end{cases}
$$

The only exception is when the values of $\tau_{p q}^{b}$ and $y_{p q}^{i j}$ are both 1 , in which case the value of ${ }^{b} \eta_{p q}^{i j}$ should be 1 as well to keep consistency. This is guaranteed by constrain (ii) of equation (4).

The optimization problem resulting from merging equation (1), equation (2), equation (3) and equation (4), express the problem MORNDP through a MIP (Mixed Integer Programming) formulation.

\section{COMPLEXITY AND EXACT SOLUTIONS}

Until now we expressed the problem through two layers. Although this is an accurate approximation of what really happens, we could go forward and think about this problem as a three layers model. 


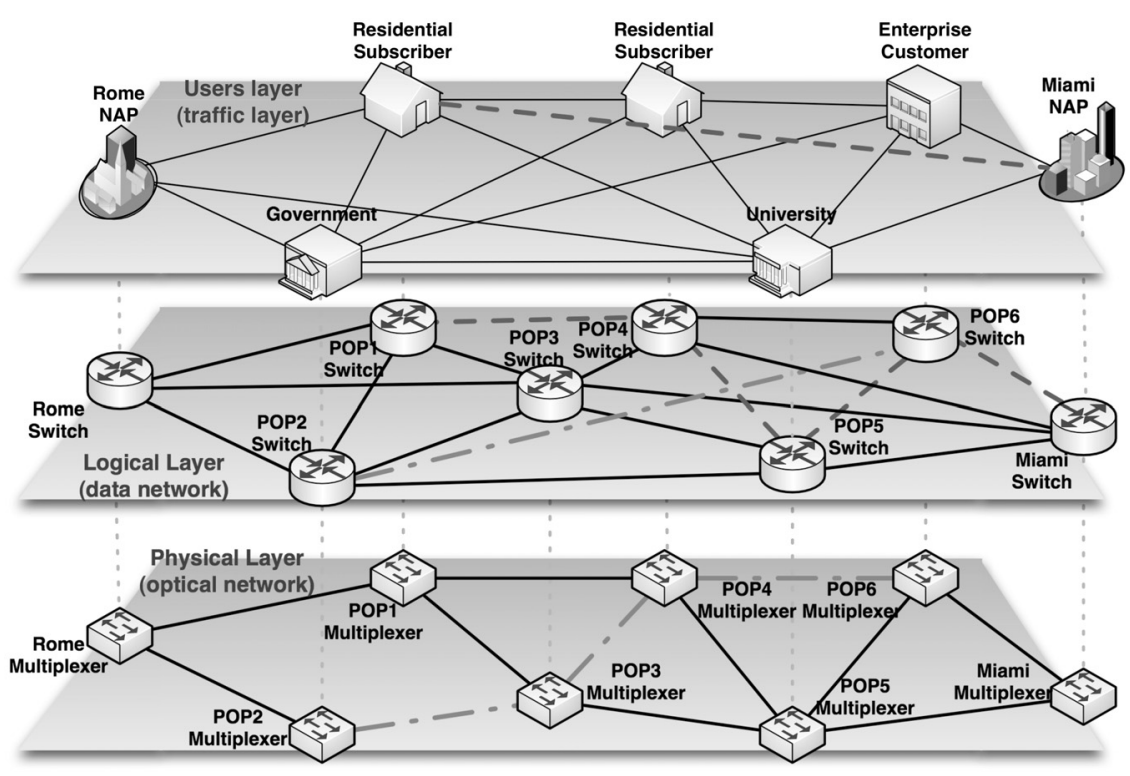

Figure 1 - A three layers conceptual model.

Besides the formerly defined logical and physical layers, there is another abstract overlay on the top of them "the service perspective about the problem" or traffic layer, i.e., how are logical paths arranged to fulfill point-to-point demands between nodes (Figure 1).

Traffic volume is a primary component of the input data-set, which impacts deeply in the shape of the result.

\subsection{Complexity}

Since in this problem the traffic between nodes cannot be split into more than one path, the task of arranging tunnels over the logical layer is a remarkable subtask, closely related to - and basically as hard as - Integer Knapsack or Number Partition Problem (NPP). These are both well known NP-Complete problems. Hence, the shape of the traffic layer directly impacts in the complexity of the problem instance.

In [15], the NP-Completeness of the problem MORNDP was based on the interaction between traffic and logical layers. That is, the mere routing of the IP/MPLS tunnels over the Data Network - even without considering faulty physical links - is NP-Hard. The proof into the referenced article is based on a polynomial reduction of NPP to MORNDP.

In this article we present an alternate proof based on the interaction between logical and physical layers. This complementary proof has the extra value to show that the mathematical program detailed in Section 3 (MORNDP) is indeed the composition of solely very hard to solve problems.

Proposition 4.1. The problem MORDNP is NP-Hard. 
Proof. Proof lies under reduction of the 2ECSS problem (Two-Edge-Connected Spanning Subgraph) to our particular problem that we will refer to as MORNDP (Multi-Overlay Resilient Network Design Problem). The 2ECSS problem consists in finding a minimum-weight subgraph of a weighted graph, in which every pair of vertices is two-edge-connected. The 2ECSS problem is a very well known NP-Hard problem (see for instance [2]).

We will use decision versions of both problems to validate instances. Let $\pi_{k}$ be the decision problem consisting in saying whether it exists a feasible solution for MORNDP of cost $k$, so that for every $k^{\prime}<k$ the answer to $\pi_{k^{\prime}}$ is negative.

Analogously, let $\pi_{k}^{\prime}$ be the decision problem consisting in saying whether it exists a feasible solution for the 2ECSS problem of cost $k$, so that there is not other solution of lower weight.

$(\Rightarrow)$ Given an instance $G=(V, E, W)$ of the 2ECSS problem - for simplicity we will assume that all the weights are positive integers - we create an instance of MORNDP by taking $\hat{B}=\{N(N-1) / 2\}, N=|V|$, logical and physical graphs with the same topology of $G$, $l_{i j}=w_{i j} \forall(i j) \in P$ (weights of $G$ arcs) and $d_{i j}=1, \forall 1 \leq i<j \leq N . c_{b}=1$ for the unique available capacity.

If the given instance satisfies the decision problem $\pi_{k}^{\prime}$, is because there exists $G^{\prime} \subseteq G$ of weight $k$ so that $G^{\prime}$ is two-edge-connected and there is not other solution of lower weight.

The rules to construct a solution for $\pi_{k}$ from $G^{\prime}$ are the following:

1. Take every edge of $G^{\prime}$ and dimension its homologous logical link with capacity $b_{1}$.

2. The remaining logical links will not be used.

3. Implement every effective logical link using its associated physical link.

It is immediate that the cost of the previous solution is $k$. Complementarily, logical link failures are one-to-one with physical link ones and therefore single physical failures affect at most one logical link. Due to the fact that logical layer copies the topology of $G^{\prime}$, the logical layer remains connected against any single physical link failure.

Finally, the capacity used in the logical links guarantees that none can be saturated. Hence, due to the fact that the construction mechanism turns connectivity into feasibility, the solution proposed for $\pi_{k}$ is feasible.

It suffices to observe that it cannot exist another feasible solution for $\pi_{k}$ of lower cost, because that would imply that it also exists a lower cost solution for $\pi_{k}^{\prime}$. The formal arguments are basically the same ones used in the following part of the proof.

$(\Leftarrow)$ First, we claim that given any solution to a positive instance of $\pi_{k}$ constructed by the previous mechanism from some $\pi_{k}^{\prime}$, it holds that its lightpaths are edge-disjoint. Indeed, suppose that $(i j)$ and $\left(i^{\prime} j^{\prime}\right)$ were two logical links whose lightpaths intersect, like in Figure 2. Then, another solution can be built by exchanging $(i j)$ and $\left(i^{\prime} j^{\prime}\right)$ by the logical links associated to their lightpaths implementations, like it is sketched in the second half of Figure 2. 


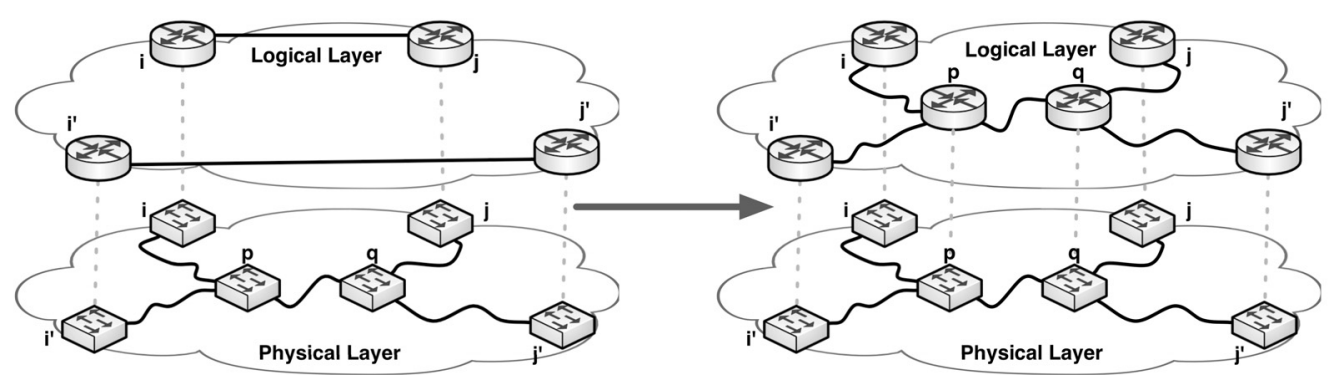

Figure 2 - Non-disjoint lightpaths implementation and an alternate configuration.

If during the transformation we found that one of the new logical links is already present, we just skip this particular appending.

The transformation preserves - may even surpass - the logical connectivity in every failure scenario. It is also cheaper because the physical links between $p$ and $q$ are only used once in this solution. The last feature cannot hold because it would exist a solution whose cost is lower than $k$, which is explicitly forbidden in this decision problem. This ends the proof of our claim.

As a corollary of the previous property, we can prove that given any solution to a positive instance of $\pi_{k}$ is always possible to build an equivalent one where topologies of logical and physical layers match. The transformation process is based on the following recurrence:

1. If any effective logical link is not implemented using its corresponding physical link, we must replace it with the logical links associated to the links of the lightpath.

2. If during the previous step a logical link is repeated, the former is replaced by the logical links associated to the links of its lightpath. This can always be done because implementations must be disjoint.

3. Repeat the process until logical and physical networks match.

Since this transformation preserves the usage of links in the physical network, the cost of the solution and the failure scenarios are not affected.

Remains to be seen that the topology found $\left(G^{\prime}=\left(V, E^{\prime}\right)\right)$ is Two-Edge-Connected, so it is also a solution to $\pi_{k}^{\prime}$. Certainly, whenever a physical link $l \in E^{\prime}$ fails the remaining operational links of the logical layer $\left(E^{\prime} \backslash\{l\}\right)$ must be capable of delivering the traffic. But since there is traffic demand between each pair of nodes, this is equivalent to state that $\forall l \in E^{\prime}, G^{\prime} \backslash\{l\}$ is connected, so it is immediate that $G^{\prime} \subseteq G$ is a two-edge-connected graph.

Finally, it cannot exist another feasible solution to $\pi_{k}^{\prime}$ of cost $k^{\prime}<k$, because the outcome of the transformation described in $(\Rightarrow)$ to this solution would be a feasible solution to $\pi_{k}$ of cost lower than $k$. Let us keep in mind that the construction process turn connectivity into feasibility.

Since all the transformation are of a polynomial complexity it stands that $\pi_{k}^{\prime} \preccurlyeq \pi_{k}$, and due to the fact that the 2ECSS problem is NP-Hard, MORNDP is NP-Hard too. 
Although finding optimal solutions to this problem is usually very hard to accomplish, discarding them may be much easier. The following necessary condition proved to be a very useful tool for that.

\subsection{Bonds and necessary condition}

A "bond" is a well-known part of the graph theory glossary. It is called so to any minimal (but not necessarily minimum), proper, not empty set of edges whose removal disconnects the graph, splitting it into two components. We will extend this definition to our problem:

Definition 4.2. Let MORNDP be the problem presented in Section 3. Any bond $P$ (regular bond of the physical layer), will also be called a "bond of MORNDP" when: being $\left(V^{\prime}, P^{\prime}\right)$ and $\left(V^{\prime \prime}, P^{\prime \prime}\right)$ the two connected components of $(V, P) \backslash$ bond $_{P}$, it holds that: $\left(V^{\prime}, L \cap V^{\prime 2}\right)$ and $\left(V^{\prime \prime}, L \bigcap V^{\prime \prime 2}\right)$ are both connected components too. For simplicity we will refer to $L \bigcap V^{\prime 2}$ and $L \bigcap V^{\prime \prime 2}$ as: $L^{\prime}$ and $L^{\prime \prime}$ respectively.

We will also define bond b,$P=\left\{e \in L / e=p q, p \in V^{\prime}, q \in V^{\prime \prime}\right\}$, which is actually a regular bond of $(V, L)$ induced by bond $P$.

The following property proved to be very useful during our algorithm implementation because it stands a very simple way to discard solutions.

Lemma 4.3. Let MORNDP be a problem as presented in Section 3. Given any solution to this problem, let $\bar{L} \subseteq L$ be the subset of arcs assigned with positive capacities. In order to be feasible, this solution must satisfy the following condition for every possible bond $d_{P}$ :

$$
\sum_{p \in V^{\prime}, q \in V^{\prime \prime}} d_{p q} \leq b_{\bar{B}}\left\lfloor\frac{\mid \text { bond }_{\bar{L}, P} \mid\left(\mid \text { bond }_{P} \mid-1\right)}{\mid \text { bond }_{P} \mid}\right\rfloor
$$

Proof. The proof is based on a particular case of the "Pigeonhole Principle". This is because regardless of how links in bond $_{\bar{L}, P}$ are implemented over the physical layer, they must use some link of bond $_{P}$ to connect $\left(V^{\prime}, P^{\prime}\right)$ with $\left(V^{\prime \prime}, P^{\prime \prime}\right)$. Then, there must exist at least one physical edge $e \in$ bond $_{P}$, used at least $\left\lceil\mid\right.$ bond $_{\bar{L}, P}|/|$ bond $\left._{P} \mid\right\rceil$ times by lightpaths of bond $_{\bar{L}, P}$ (Pigeonhole Principle).

As a consequence, if the physical link $e$ fails, the remaining capacity to route traffic from $\left(V^{\prime}, L^{\prime}\right)$ to $\left(V^{\prime \prime}, L^{\prime \prime}\right)$, falls down to at most $b_{\bar{B}}\left(\mid\right.$ bond $_{\bar{L}, P} \mid-\left\lceil\mid\right.$ bond $_{\bar{L}, P}|/|$ bond $\left.\left._{P} \mid\right\rceil\right)$. If this capacity is below traffic demand between components $\left(\sum_{p \in V^{\prime}, q \in V^{\prime \prime}} d_{p q}\right)$ the logical network cannot satisfy demands. The previous condition it is numerically equivalent to state that if

$$
\sum_{p \in V^{\prime}, q \in V^{\prime \prime}} d_{p q}>b_{\bar{B}}\left\lfloor\frac{\mid \text { bond }_{\bar{L}, P} \mid\left(\mid \text { bond }_{P} \mid-1\right)}{\mid \text { bond }_{P} \mid}\right\rfloor
$$

there is always an edge $e \in$ bond $_{P}$, used so many times, that its fault leaves the resultant operational data network without enough capacity in $\operatorname{bond}_{\bar{L}, P}$ to route traffic between nodes of $V^{\prime}$ and $V^{\prime \prime}$. 
It is worth mentioning that the previous property is a generalization of the method given in [9]. For instance, in Figure 3 when $b_{\bar{B}}=1 \mathrm{Gbps}$, the best bandwidth scenario between components is that where all links are assigned with 1 Gbps (i.e.: $\bar{L}=L$ ).

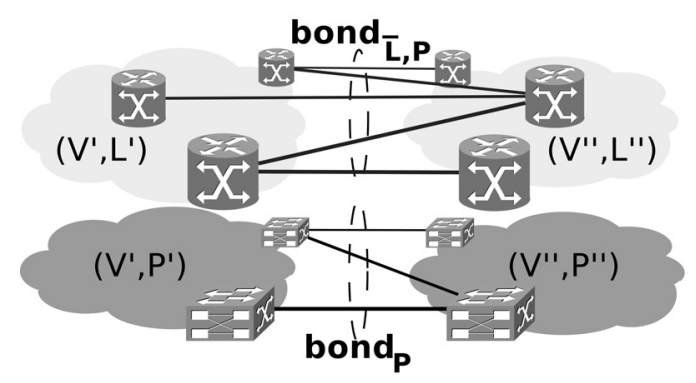

Figure 3 - Extended version of Bonds to problem MORNDP.

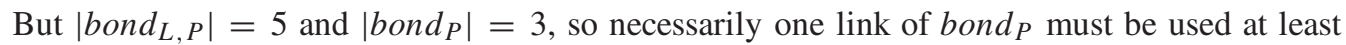
twice to implement bond $_{L, P}$ lightpaths. If this link fails, the remaining capacity between $\left(V^{\prime}, L^{\prime}\right)$ and $\left(V^{\prime \prime}, L^{\prime \prime}\right)$ falls to at most 3 Gbps. If aggregated traffic requirements between components were above this value, the solution could not be feasible.

The condition is not sufficient as we show below.

\subsubsection{Counterexample to the bond condition}

Consider the following problem: $V=\left\{v_{1}, v_{2}, v_{3}, v_{4}\right\}, P=L=\left\{\left(v_{1} v_{2}\right),\left(v_{2} v_{3}\right),\left(v_{3} v_{4}\right)\right.$, $\left.\left(v_{4} v_{1}\right),\left(v_{1} v_{3}\right)\right\}, \hat{B}=\{1\}$ and $d_{13}=d_{24}=1$. Physical and Logical topologies are represented in the left side of Figure 4, while the second half sketches the traffic demands.
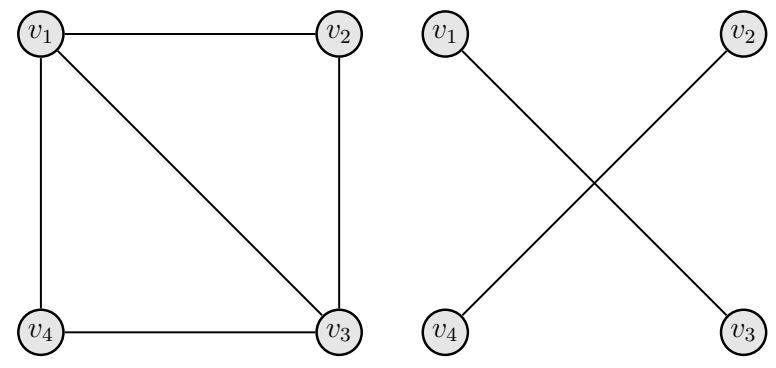

Figure 4 - Counterexample to bonds conditions.

Because of symmetry, $\left(\left\{v_{1}\right\},\left\{v_{2} v_{3} v_{4}\right\}\right),\left(\left\{v_{2}\right\},\left\{v_{1} v_{3} v_{4}\right\}\right)$ and $\left(\left\{v_{1} v_{2}\right\},\left\{v_{3} v_{4}\right\}\right)$ comprise the representative failing scenarios for bonds. Let us start by checking out that equation (5) is satisfied for these scenarios:
1) $1 \leq 1\left\lfloor\frac{3(3-1)}{3}\right\rfloor=2$
2) $1 \leq 1\left\lfloor\frac{2(2-1)}{2}\right\rfloor=1$,
3) $2 \leq 1\left\lfloor\frac{3(3-1)}{3}\right\rfloor=2$. 
The bond condition is always satisfied.

Suppose that some candidate solution is given for this instance. Regardless of how lightpaths are implemented, there must be some physical link whose fault affects logical link $v_{1} v_{3}$ lowering down the operational logical layer to at most $C^{4}$. Since traffic from $v_{1}$ to $v_{3}$ and from $v_{2}$ to $v_{4}$ must cross each other into some link and $\hat{B}=\{1\}$, the reduced logical networks cannot satisfy the demands.

This condition assists the analysis of candidate solutions but stronger results were needed to construct the solutions themselves.

\subsection{Exact solutions}

Because of the complexity of the problem it is not expectable to find analytic solutions for the general case. Despite that, the analysis of particular cases was a key factor since many practical results were obtained from it.

Planarity is a common characteristic to most optical fiber networks, because most of them are deployed over earth's surface.

Due to the fact that most physical networks can be decomposed into faces, we aimed to find analytic solutions for this case as a foundation, over which construct feasible solutions. The following results correspond to limit cases of this kind of structure.

Theorem 4.4. Given $\mathcal{K}^{n}$ (clique) and $C^{n}$ (cycle) respectively as logical and physical layers, and if demands conform to $d_{p q} \leq D$, is always possible to find minimal feasible solutions for MORNDP when $b_{\bar{B}}=2 D$ and $n$ is odd, or when $b_{\bar{B}}=3 D$ and $n$ is even.

Moreover, the solution when $d_{p q}=D$ and $n$ is odd requires the usage of all the links of $\mathcal{K}^{n}$, whereas if $n$ is even only diagonal links can be discarded, except for $n=4$.

Proof. This proof has several steps. First of all let us observe that $d_{p q}=D$ is the hardest demand case, so proving that if $d_{p q}=D$ and $n$ is odd, the entire $\mathcal{K}^{n}$ with links dimensioned with a capacity $2 D$ is an optimal solution for the logical layer, would be enough for the feasibility (if $d_{p q}<D$ ) and the optimality (when $d_{p q}=D$ ) for the odd case. Analogously, for $n$ even we will prove that $\mathcal{K}^{n}$ minus diagonals links, dimensioned with a capacity $3 D$ is optimal.

The next part of the proof consists in determining lower bounds for the capacity $b_{\bar{B}}$ and the number of logical links to implement when $d_{p q}=D$. After that we will show how to construct a feasible solution using that bounds. The minimal nature of that construction will close the optimality of the solution.

To determine lower bounds we will apply Lemma 4.3 using the bond defined by any node (e.g. $\left.v_{1}\right)$ to its complement. Since we are seeking lowest values for $b_{\bar{B}}$ the maximum degree of logical connectivity will be allowed. Since $v_{1}$ has 2 neighbors in the physical layer and $(n-1)$ neighbors in the logical one, using Lemma 4.3 we have that $D(n-1) \leq b_{\bar{B}}\lfloor(n-1) / 2\rfloor$. If $n$ is odd this 
inequality turns to $D(n-1) \leq b_{\bar{B}}(n-1) / 2$ and $b_{\bar{B}}$ must satisfy $b_{\bar{B}} \geq 2 D$ in order to guarantee the existence of solutions.

On the other hand, if $n$ is even the inequality turns to $D(n-1) \leq b_{\bar{B}}(n-2) / 2$ and $b_{\bar{B}}$ must satisfy $b_{\bar{B}} \geq 2 D(n-1) /(n-2)$. Since traffic between each pair of nodes is $D$ and our problem does not allow to split this traffic through more than one path (tunnel), the capacity of any logical link has to be an integer multiple of $D$ to be useful. In other words, none of the tunnels can fit within a fraction of $D$. The first integer multiple of $D$ greater or equal to $2 D(n-1) /(n-2)$ is $3 D$, so practical solutions actually require that $b_{\bar{B}} \geq 3 D$.

It is time now to prove that those boundaries allow building feasible solutions. If $n=3$ then $\mathcal{K}^{n}$ matches $C^{n}$ links of both layers one-to-one. Hence, whenever a link fails, the only way to deliver traffic is through the link with the remaining logical neighbor, and capacity $2 D$ is enough for that.

The case $n=4$ is slightly tricky. The first half of Figure 5 shows the best choice for the path of each lightpath. The failure scenarios that affect most links are $\left(v_{1} v_{4}\right)$ and $\left(v_{2} v_{3}\right)$, reducing the logical layer to what is sketched in the second half of Figure 5.
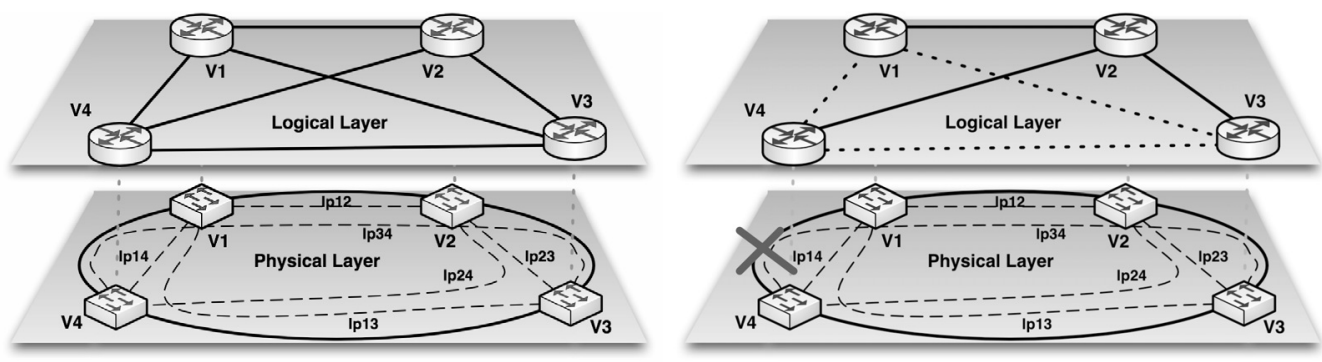

Figure 5 - Optimal solution found for $\mathcal{K}^{4}$ over $C^{4}$, with $d_{p q}=D$ and $B=\{3 D\}$.

For this path configuration and if $b_{\bar{B}}=3 D$ the solution is feasible too. Actually, this configuration was found with CPLEX as the optimal solution for the set of parameters.

For values of $n$ greater than 4 a more general rule must be used. Let us suppose that $n>4$ and odd, so $n=2 k+1$. The proposed implementation uses all of the logical links $\left(\mathcal{K}^{n}\right)$ and the shortest number of physical hops to implement the lightpath of each one. Since $n$ is odd this implementation is unique for each lightpath.

To route traffic between nodes in the non-faulty state, the direct route may be used - all nodes are neighbors -. For simplicity we will assume that nodes are numbered like $\left(v_{0}, \ldots, v_{2 k}\right)$. Due to the fact that $C^{n}$ and $\mathcal{K}^{n}$ have cyclic symmetry, we can analyze a particular failure without loss of generality. Let $\left(v_{0} v_{2 k}\right)$ be the physical link in faulty state. This fault condition affects all logical links of the form $\left(v_{i^{\prime}} v_{i^{\prime \prime}}\right)$ where:

$$
\left.\begin{array}{l}
i^{\prime}=0,1, \ldots, k-1 \\
i^{\prime \prime} \in\left\{k+i^{\prime}+1, \ldots, 2 k-1,2 k\right\}
\end{array}\right\}
$$


On the first half of Figure 6 we represented an example of the effects of this fault over the logical layer, for $n=5(k=2)$.
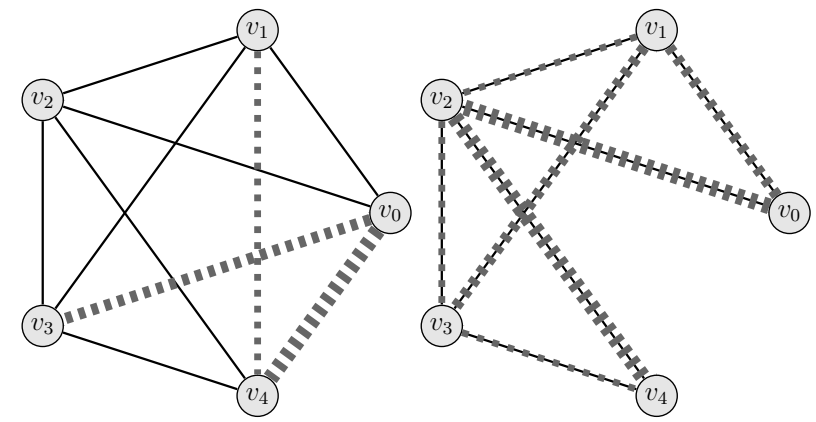

Figure 6 - Example case for $n=5$.

Dashed lines represent affected links. For those tunnels not affected by the fault, the direct logical route will be preserved. The surviving logical links still have a gap of capacity of magnitude $D$, and we must find a way to deploy affected tunnels over them. The strategy is the following: take affected nodes in decreasing order of severity and attempt to detour all its affected tunnels in the minimum number of hops. This can be accomplished in 2 hops for $v_{0}$ - the most affected one and in 3 hops for the remaining nodes.

The alternative route for those tunnels that followed a path of the form $\left(v_{0} v_{i^{\prime \prime}}\right)$ now turns to $\left\{\left(v_{0} v_{i^{\prime \prime}-k}\right),\left(v_{i^{\prime \prime}-k}, v_{i^{\prime \prime}}\right)\right\}, i^{\prime \prime}=k+1, \ldots, 2 k-1,2 k$. All the remaining faulty tunnels will be detoured in 3 hops according to the following rule: a faulty tunnel $\left(v_{i^{\prime}} v_{i^{\prime \prime}}\right)$ will follow the path $\left\{\left(v_{i^{\prime}} v_{i^{\prime \prime}-k}\right),\left(v_{i^{\prime \prime}-k}, v_{i^{\prime \prime}-i^{\prime}}\right),\left(v_{i^{\prime \prime}-i^{\prime}}, v_{i^{\prime \prime}}\right)\right\}, i^{\prime \prime}=k+i^{\prime}+1, \ldots, 2 k-1,2 k$.

This new configuration is also sketched in Figure 6, where different kinds of dashed lines in the second diagram correspond to the route of the associated faulty tunnel.

To be sure this configuration is feasible, we must check that logical links are operational in this state and none is used more than once. Both are straightforward:

- Since all links are forward links with a number of hops less or equal than $k$, by construction they cannot use $\left(v_{0} v_{2 k}\right)$ and must stand operational.

- The first logical link used to detour $\left(v_{0} v_{i^{\prime \prime}}\right)$ always starts at $v_{0}$, while the second leap always has $k$ hops. Since none of the links for the 3 leaps group has such length or origin, both sets cannot intersect.

- Within each set, logical links cannot be repeated by construction.

We used lower bounds to dimension logical links and wasted all of them, so this feasible solution is minimal and must be optimal, regardless of the lengths of the physical layer.

The final step of this proof is for $n$ greater than 4 and even. Let us say $n=2 k$. As it was seen, $b_{\bar{B}}=3$ is a lower bound for the capacity so we will attempt to use this value as a starting one. 
Unlike the $n$ odd case, in this case, we opted for deleting diagonal links of the logical layer. This decision keeps unique the election of the lowest number of hops to implement lightpaths. The remaining aspects of the construction stay equal, except for some paths followed by the tunnels within the logical layer.

Since in the this construction we cannot use diagonals, the traffic between $v_{i}$ and $v_{i+k}(0 \leq$ $i \leq k-1)$ will be routed through $\left\{\left(v_{i} v_{i+k-1}\right),\left(v_{i+k-1}, v_{i+k}\right)\right\}, i=0, \ldots, k-1$. This leaves a remaining capacity of 2 or 3 in all of the logical links.

Instead of going into details of the construction of the tunnels within the logical layer in the representative fault state $\left(v_{0} v_{2 k-1}\right)$, we will present an illustrative workaround.
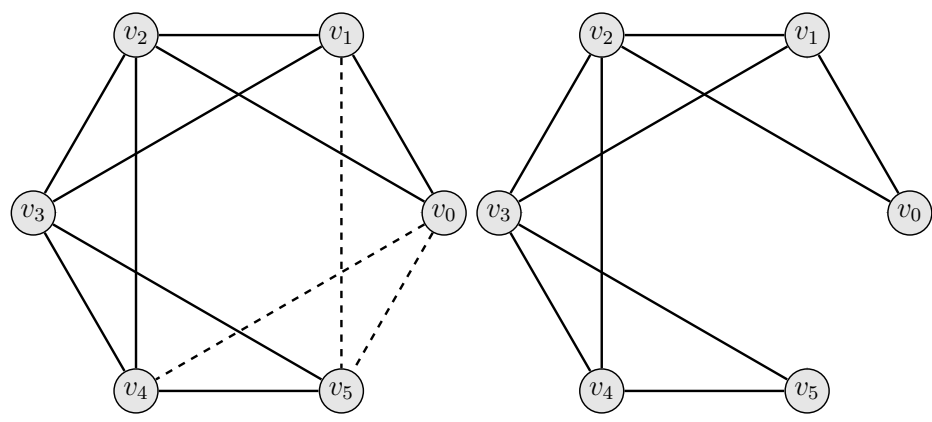

Figure 7 - Example case for $n=6$.

Figure 7 outlines in its first half a diagram for the logical layer when $n=6$, and represents with dashed lines the logical links affected by the failure of the physical link $\left(v_{0} v_{5}\right)$. The second half of the picture details the operational logical links in this faulty state. If instead of this figure we look back to the second picture of Figure 6, we might conclude that both graphs look very similar. In fact, besides $v_{5}$ they only differ in links $\left(v_{3} v_{5}\right)$ and $\left(v_{4} v_{5}\right)$. Moreover, in both cases demand between $v_{0}$ and $v_{4}$ must be reestablished, but instead of demands between $v_{0}$ and $v_{3}$, and between $v_{1}$ and $v_{4}$, in this case we have to figure out a solution to detour tunnels for pairs $\left(v_{0}, v_{5}\right)$ and $\left(v_{1}, v_{5}\right)$. An easy workaround would be to use the former solution for $n=5$ and adapt it to the new case. This can be achieved appending $\left(v_{3} v_{5}\right)$ to the known tunnel between $v_{0}$ and $v_{3}$, and appending $\left(v_{4} v_{5}\right)$ to the known tunnel between $v_{0}$ and $v_{4}$. The previous ideas can be easily generalized for any $k>6$ and even.

Corollary 4.1. It is always possible to find minimal feasible solutions when:

1. The logical layer is $\mathcal{K}^{n}$;

2. The physical layer is a 2-edge-connected graph $G$ (on the $n$ vertices of the logical layer), such that each one of its $k$ blocks is a cycle;

3. The demands conform to: $d_{p q} \leq D$;

4. Capacities comply either $b_{\bar{B}}=3 D$ when $n$ is even or $b_{\bar{B}}=2 D$ when $n$ is odd. 
Proof. First of all, let us notice that any graph $G$ in the condition of 2 ), is made up by identifying $k-1$ vertices from a cycle of $n+k-1$ vertices. These vertex identifications originate the cut-vertices of $G$.

An example instance of such $G$ is represented on the right of Figure 8. A cycle, which can generate this instance after a selective identification process (marked with thin dashed lines), is sketched on the left half of the same figure. Within this proof, we refer to such a cycle with the list of identifications as an expansion of $G$.
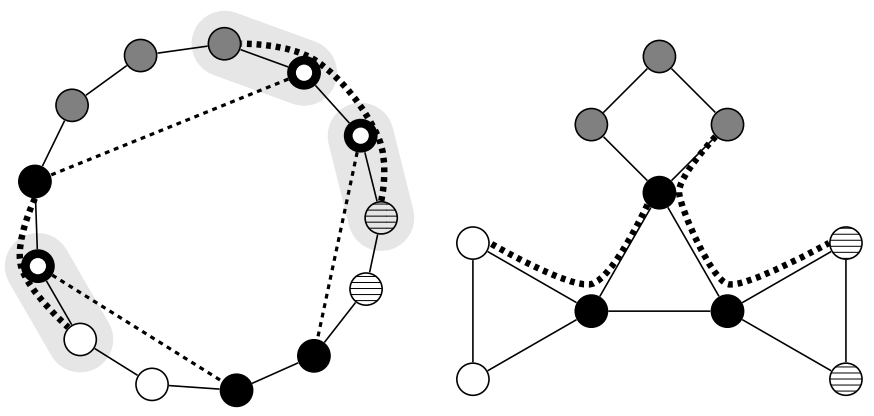

Figure 8 - Example case for $n=10, k=4$.

It is tempting the idea of simply applying Theorem 4.4 over the expanded $C^{n+k-1}$. However, this cannot be done directly because nodes upon both layers do not match after expansion. In other terms only one on each set of identified physical nodes can have an associated logical node. This can nonetheless be fixed easily by contracting $k-1$ nodes. The first step consists in assigning - arbitrarily - the logical node corresponding to each expanded set to one of the physical nodes. The remaining $k-1$ logically unpaired nodes are recursively contracted with neighbors in $C^{n+k-1}$ until get to $C^{n}$.

For instance in Figure 8 those nodes without a logical peer are marked with a white dot into the middle. Besides, in this example, those nodes selected for being contracted are shadowed with pale gray.

Once in this state we can apply Theorem 4.4 construction to determine capacities and routing configurations on both layers. To roll back this configuration over $C^{n+k-1}$ we need to reinsert those edges contracted that would be over the path. This is represented on Figure 8 using bolddashed black curves.

Since edges of $C^{n+k-1}$ after identifying the corresponding vertices, are the same edges of $G$, a failure in any edge of $G$ produce the same effect than in the corresponding edge of $C^{n+k-1}$. Therefore replicating paths over the logical layer following the per-scenario path configuration of each LSP found for Lemma 4.4 works fine in this graph also.

The previous results are very helpful to design within physical faces highly populated with logical links; a situation that arises when demands overflow links capacity. On the other hand, there is another interesting property for the simplest possible case. 
Proposition 4.5. Given $C^{n}$ as the physical layer and any logical layer $L$ so that $C^{n} \subseteq L$, if demands conform to $d_{p q} \leq D$ it is always possible to find minimal feasible solutions to MORNDP when $b_{\bar{B}}=D n^{2} / 4$ and $n$ is even, or if $b_{\bar{B}}=D\left(n^{2}-1\right) / 4$ and $n$ is odd.

Moreover, the solution in both cases reduces to use $C^{n}$ as the logical layer.

Proof. The proof in this case relays on finding the most critical bond for the solution. Let us take $C^{n}$ as the effective topology for the logical layer, where all links are dimensioned with capacity $b_{\bar{B}}$. We will prove the feasibility for the hardest case (i.e. $d_{p q}=D$ ).

If $n$ is even we will take bond $_{P}=\left\{\left(v_{1} v_{n}\right),\left(v_{n / 2} v_{n / 2+1}\right)\right\}$. This bond splits $V$ into $V^{\prime}=$ $\left\{v_{1}, \ldots, v_{n / 2}\right\}$ and $V^{\prime}=\left\{v_{n / 2+1}, \ldots, v_{n}\right\}$. Let us observe that $\mid$ bond $_{P}|=|$ bond $_{\bar{L}_{, P}} \mid=2$. Applying Lemma 4.3 to this instance it gets that:

$$
\sum_{p \in V^{\prime}, q \in V^{\prime \prime}} D=\sum_{p=1}^{n / 2} \sum_{p=n / 2+1}^{n} D=\left(\frac{n}{2}\right)^{2} D \leq b_{\bar{B}}
$$

If $n$ is odd we will take bond $_{P}=\left\{\left(v_{1} v_{n}\right),\left(v_{(n-1) / 2} v_{(n+1) / 2}\right)\right\}$. This bond splits $V$ into $V^{\prime}=$ $\left\{v_{1}, \ldots, v_{(n-1) / 2}\right\}$ and $V^{\prime \prime}=\left\{v_{(n+1) / 2}, \ldots, v_{n}\right\}$. Again in this case $\mid$ bond $_{P}|=|$ bond $_{\bar{L}, P} \mid=2$, but now Lemma 4.3 turns to

$$
\sum_{p \in V^{\prime}, q \in V^{\prime \prime}} D=\sum_{p=1}^{(n-1) / 2} \sum_{p=(n+1) / 2}^{n} D=\frac{(n-1)}{2} \frac{(n+1)}{2} D=\frac{\left(n^{2}-1\right)}{4} D \leq b_{\bar{B}}
$$

Given $n$ we will take the corresponding limits as the value for $b_{\bar{B}}$. Due to the low connectivity of this topology each logical link must to be implemented using its associated physical link, otherwise lightpaths would intercept in some physical link and the logical network would be disconnected by its fault.

We only need to check now that this implementation is feasible. If $n$ is even and some physical link fails, the logical layer reduces to a sequence of connected nodes. Without loss of generality, the arcs of the logical layer could be $\left\{\left(v_{1} v_{2}\right),\left(v_{2} v_{3}\right), \ldots,\left(v_{n-1} v_{n}\right)\right\}$. Given any of these arcs $\left(v_{k}, v_{k+1}\right), 1 \leq k<n$, the traffic across this link would be

$$
\sum_{1}^{k} \sum_{k+1}^{n} D=k(n-k) D \text {. }
$$

If $n$ is even this function takes its maximum at $n / 2$ which image is $D n^{2} / 4$, the value chosen to dimension links. If $n$ is odd this function takes its maximum at $(n-1) / 2$ and $(n+1) / 2$ which image is $D\left(n^{2}-1\right) / 4$, and also matches the dimension of the logical link.

Intermediate cases are much harder to analyze. It is worth mentioning that our team used CPLEX to find solutions, but even over these trivial topologies we couldn't find solutions for $n \geq 10$ when $d_{p q}$ was constant, or for $n \geq 15$ when $d_{1 q}$ was constant and $d_{p q}=0$, forall $p, q$, such that $1<p<q \leq n$. Best computation times - by far - were associated to limit cases of Theorem 4.4 and Proposition 4.5. 


\section{A REAL-WORLD APPLICATION CASE}

In order to find solutions for real applications we used metaheuristics, but before going any further we will present some aspects of the concrete problem of ANTEL.

\subsection{Context and characteristics}

Internet is actually a network disaggregated into several separate smaller networks also known as Autonomous Systems (AS). Typically, every AS is a portion of the global Internet owned/ governed by a particular Internet Service Provider (ISP). Network nodes and content servers of ISPs are geographically distributed over POPs (Point-of-Presence).

Internet users access content residing in servers of companies, universities, government sites or even from other residential customers (e.g. P2P applications). Most of these servers as well as servers of Content Delivery Networks (CDN) - e.g. Google or Akamai - are installed into datacenters. Since traffic interchange is necessary among different ISPs, the Internet architecture needs special POPs known as Network Access Points (NAPs).

The previous high-level structure is shared among ISPs all over the world; additional details are proper to the design criteria established by each one. Regarding our application case, ANTEL had two different IP/MPLS networks referred to as aggregation network and public Internet network. The aggregation network is geographically dispersed all over the country and it is responsible of gathering and delivering the traffic of the customers, to the public Internet network.

The public Internet network is where the AS of this ISP is implemented; it centralizes the international connections with other ISPs as well as those to Datacenters of local content providers. The public Internet network is geographically concentrated and only has POPs in the Capital City and in an important NAP of the US territory (see grey clouds in Figure 9).

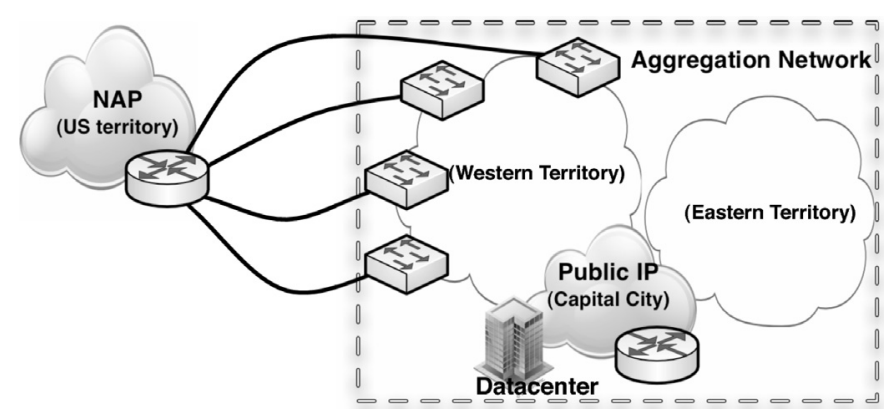

Figure 9 - Structure of the particular network architecture.

In terms of the model covered in this article we may stand that the physical network has all of its nodes but one - the NAP - within the national boundaries; the aggregation and public Internet networks are both logical. The public Internet network only has presence in a few POPs of the Capital City (Montevideo) and in the NAP; and although the aggregation network has full-national presence it does not span to the NAP. 
Several planning concerns arose from the situation exposed:

- Is the current architecture convenient? or Would it be better to merge both IP/MPLS networks?

- Are the IT infrastructure investments necessary to increase the percentage of local content profitable?

- Which would be the optimal network to fulfill every demand requirement at lowest cost possible?

- How much is the sensitivity cost due to changes in demands?

To answer these questions we identified representative scenarios and used metaheuristics to find good quality solutions for them. Scenarios were selected varying the following factors: traffic volume, network architecture and the percentage of locally terminated traffic. We selected eight remarkable scenarios as detailed in Table 1. The costs and traffic information shown in the rest of this article are only referential.

According to the traffic forecasts, it is expected that in a few years, the total volume of traffic would be placed somewhere between 56 and 100 (reference values). Additionally, if some IT investments and agreements were made, it is expected that the percentage of locally terminated traffic (national traffic) could be greater (High). Both factor were considered to determine scenarios.

Table 1 - Referential results for representative scenarios.

\begin{tabular}{|c|c|c|c|c|c|c|}
\hline $\begin{array}{c}\text { scenario } \\
\text { index }\end{array}$ & $\begin{array}{c}\text { aggregated } \\
\text { traffic demand }\end{array}$ & $\begin{array}{c}\text { \% local } \\
\text { content }\end{array}$ & $\begin{array}{c}\text { merged } \\
\text { networks }\end{array}$ & $\begin{array}{c}\text { number } \\
\text { of nodes }\end{array}$ & $\begin{array}{c}\text { required } \\
\text { lightpaths }\end{array}$ & $\begin{array}{c}\text { total } \\
\text { cost }\end{array}$ \\
\hline 1 & 100 & Low & False & 56 & 81 & $10,000,000$ \\
2 & 100 & Low & True & 68 & 133 & $7,662,651$ \\
3 & 100 & High & False & 56 & 81 & $7,578,234$ \\
4 & 100 & High & True & 68 & 118 & $5,713,563$ \\
5 & 57 & Low & False & 56 & 75 & $6,319,470$ \\
6 & 57 & Low & True & 63 & 105 & $4,872,987$ \\
7 & 57 & High & False & 56 & 75 & $5,108,587$ \\
8 & 57 & High & True & 63 & 94 & $4,064,597$ \\
\hline
\end{tabular}

Those scenarios where merged networks is set to False inherit the current network architecture two separates IP/MPLS networks -, whereas True indicates that both networks - aggregation and public Internet - have been integrated into one. In order to compare solutions fairly, the column "total cost" represents the combined cost of both networks - when they are not combined into one -. However, column "required lightpaths" only considers those of the aggregation network.

A Non-Disclosure Agreement (NDA) signed between ANTEL and our University, protects more accurate information and details. 


\subsection{Usage of Metaheuristics}

Based on the scenarios detailed in Table 1, our team explored the implementation of heuristics and metaheuristics to find answers to the former questions. The main aspects and results for them are summarized next:

1. Two Stages Approximation - Instead of attempting to optimize the entire problem at once as described in Section 3, this approximation splits the problem into two non-independent stages. The first stage aims to construct of lightpaths for the logical links, whereas the second stage focus on capacity assignments and how traffic can be routed in failure scenarios. CPLEX was used as the central optimization toolbox for this heuristic. Solutions could not be found for scenarios with merged networks (2,4,6 and 8 in Table 1). Even for the remaining scenarios, instances had to be reduced to east and west regions (see Figure 9) to find solutions.

For further information on this heuristic, see [12].

2. Genetic Algorithms - The application of sequential and parallel evolutionary algorithm to this problem showed promising results. To be practical, the model had to limit to two the number of logical routing configurations - whereas the original model allowed as many as fault scenarios - Nevertheless, with this implementation, scenarios 3, 5 and 7 could be solved.

The best solution for scenario 3 was found with this algorithm, even though the searchspace was smaller than those of the rest. For further information on the usage of this metaheuristic to the problem see [14].

3. Variable Neighborhood Search and Tabu Search - Unlike the previous examples, in this case both metaheuristics were used to optimize the problem as a whole - as described in Section 3 -. Solutions could only be found when east and west regions were treated as isolated networks. Even so, results were useful because topology depicted in Figure 9 for scenarios with separates aggregation and Internet networks (indexes 1, 3, 5 and 7), allowed to optimize each region separately as a different traffic tributary to the capital city. For further information on the usage of this metaheuristic to the problem see [1].

4. Greedy Randomized Adaptive Search Procedure - According to our benchmarks, this implementation proved to be the most suitable to find solutions for instances summarized in Table 1. Indeed, this was the only algorithm that found solutions for all scenarios, and all the solutions but one in this table, were found with GRASP. Because of its outstanding behavior we will describe this algorithm in further detail.

As a summary, for instances defined in Table 1, GRASP was the only metaheuristic capable of finding solutions on all scenarios. Moreover, in all scenarios except one, the algorithm built with GRASP has found the best solution in each case. The second best performance was achieved using Genetic Algorithms. These are the only algorithms that find solutions for the entire network, without dividing it into regions, which is not always possible on some applications. 
All algorithms were able to find solutions (optimal solutions mostly), for instances like those used in of Theorem 4.4 and Proposition 4.5. This is because of the simplicity of the physical layer on both cases. The Two Stages Approximation and VNS algorithms, hardly found solutions on physical topologies other than $C^{n}$, even for networks with less than 20 logical nodes.

\subsection{GRASP Implementation}

The Greedy Randomized Adaptive Search Procedure (GRASP), is a metaheuristic successfully applied to construct good quality solutions for several combinatorial optimization problems of diverse areas, which range from clustering ([3, 17]) to network design problems ([16]).

As for every GRASP implementation this algorithm has a loop with two phases. The construction phase builds a randomized feasible solution, from which a local minimum is found during the local search phase. This procedure is repeated MaxIter times while the best overall solution is kept as the result. Further information and details in GRASP algorithms can be found in [11, 13].

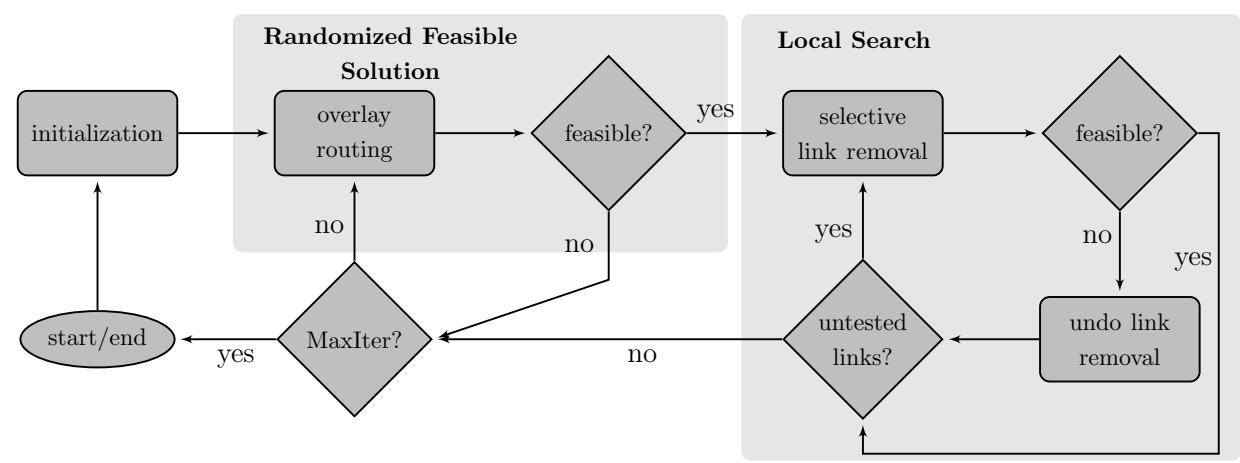

Figure 10 - Block-diagram of the GRASP implementation used.

Figure 10 outlines a block diagram of the GRASP implementation. On next subsections we analyze - phase by phase - the most relevant characteristics of this implementation. Many of them are based on the results presented in Section 4.

\subsubsection{Input data-set and initialization}

Because of strategical and economical matters, ANTEL decided to consider only 10 Gbps as a possible speed to assign to logical links. This allowed us to simplify a portion of the model described in Section 3.

According to the characteristics of each scenario some logical links must be included in the input data-set while others are forbidden. It is tempting to include all non-forbidden logical links in the input data-set, in order to count with the greatest possible search space. Unfortunately, stability and execution time of the Randomized Feasible Solution phase, degrades when too many logical links are present. 
Besides mandatory logical links, the remaining ones are included to meet constraints from Lemma 4.3, Theorem 4.4 and Proposition 4.5. For this purpose the faces of the plain representation of the physical layer are analyzed one-by-one. To determine how many logical links should be included within each face, the following rules were used:

1. Preserve traffic demands between nodes of the same face.

2. Distribute uniformly traffic terminating in nodes outside of the current face, among nodes contained both, into the current and neighbor faces.

3. Set up logical links according to Theorem 4.4 and Proposition 4.5.

For instance, Figure 11 present an hypothetical case where thin-black lines represent physical links and thick-grey ones correspond to logical internal to faces. When considering $\left\{v_{1}, v_{2}, v_{3}\right.$, $\left.v_{4}, v_{5}\right\}$, if by example $d_{17}=3$ then this demand is temporarily omitted and remaining demands are updated so: $d_{12}^{\prime}=d_{12}+1, d_{13}^{\prime}=d_{13}+1$ and $d_{14}^{\prime}=d_{14}+1$.

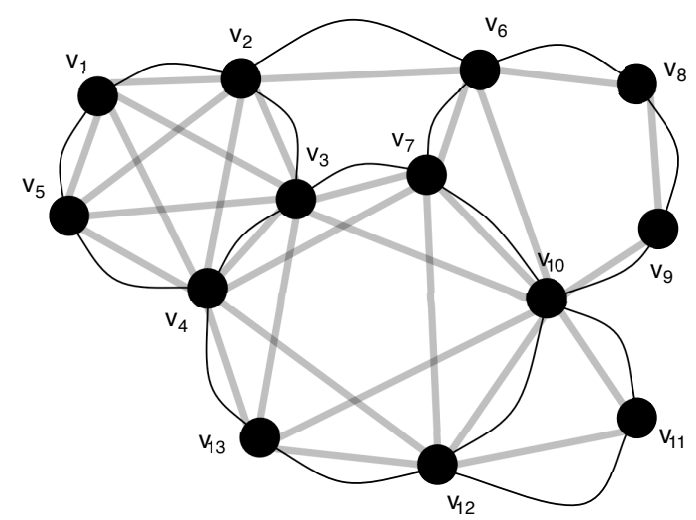

Figure 11 - Logical links internal to faces.

All internal links of the face delimited by $\left\{v_{1}, v_{2}, v_{3}, v_{4}, v_{5}\right\}$ were included because the number of nodes is odd and after computing new demands - step 2 of transformation -, some of them was close - above $60 \%$ - to $b_{\bar{B}} / 2$. A similar situation arose for $\left\{v_{4}, v_{3}, v_{7}, v_{10}, v_{12}, v_{13}\right\}$, although in this case some demand was near $b_{\bar{B}} / 3$. Diagonals were omitted because last face has an even number of nodes, as states Theorem 4.4.

In the case of faces delimited by $\left\{v_{3}, v_{2}, v_{6}, v_{7}\right\}$ and $\left\{v_{10}, v_{11}, v_{12}\right\}$, demands were below $4 b_{\bar{B}} / n^{2}$ and $4 b_{\bar{B}} /\left(n^{2}-1\right)$ respectively, corresponding to limits established in Proposition 4.5 ; then the cycle was used as the reference topology. The same situation arose for $\left\{v_{7}, v_{6}, v_{8}, v_{9}, v_{10}\right\}$ but the gap in this case was narrow. That issue, together with the fact that the number of neighbor faces for this case is above the average, led us to add an extra link $\left(v_{6} v_{10}\right)$ to facilitate detouring of traffic through this face in failure scenarios.

Besides logical links between nodes of the same face, nodes between faces were added to increase diversity of solutions. In order to do that, bond condition (equation (5)) was applied to 
several selected bonds, like for instance in Figure 12. In this case links are added until the gap between terms of equation (5) applied to the bond represented with a semi-dashed line, surpassed $50 \%$. The process is repeated for several bonds.

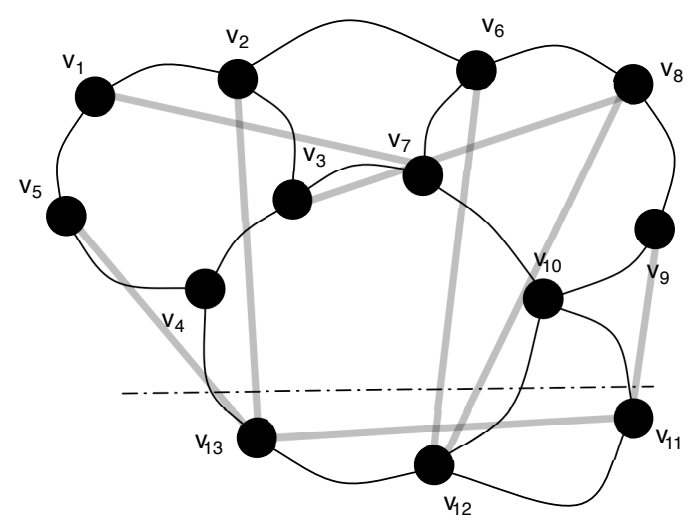

Figure 12 - Logical links between faces.

Although possible, we did not intend to automatize the previous process. The truth is that doing it manually was not a hard task and it added the value to integrate intuitive decision together with network designers, taking advantage of their experience. This work was always stated as a tool to assist decisions, not as a substitute of designers.

\subsubsection{Initializations}

The initialization phase performs computations whose results are invariants among iterations. One of them is the shortest path and distance over the physical layer between each pair of nodes.

Finding a base for structural bones is another remarkable computation realized during this phase. We apply Lemma 4.3 to increase the construction speed, discarding candidate solutions with few computations. That, would be cheap if there was an easy way to generate cycles automatically, because it is a well-known graph theory property that: "bonds of a plain graph are those whose edges form a cycle in the dual graph" (see [19]).

Unfortunately, finding all cycles within a graph, even for a planar and cubic graph, is NP-Hard. The previous is direct from [4] where it was proved that decision problem: "to find out if a planar, cubic and 3-conex graph is Hamiltonian is an NP-Hard problem".

Since in the construction of the input data-set, we were very careful to guarantee feasibility within each face, at this point we are mainly interested in bonds adjacent to borders of faces. It is through these bonds that traffic flows between nodes of different faces. Since physical degree for most nodes of the physical network was only two, we opted to a simplification of the structure before computing the dual graph. The result is still NP-Hard but since it is reduced to a bunch of nodes its complexity is manageable. 
Figure 13 shows how this idea applied to a hypothetical physical network (the left side part of the image), outcomes to a much more simple problem. The structural physical networks counts 8 nodes, whereas the original has 53 . The second half of this picture also represents the dual graph, with one node per face $\{A, B, C, D, E, F\}$ and one link between every pair of nodes whose associated faces are adjacent. The dual graph is usually a multi-graph.

Through this example example, it is easy to show how bonds are determined from cycles. For instance, there are three cycles that span nodes $\{A, C, D\}$. They are determined by the unique links between $(A, C)$ and $(C, D)$, combined with the three different links that connect $(A, D)$. Each of these cycles defines a structural bond, specifically for these cycles they would correspond to $\{$ PAY-SNR, ALG-YOU, PTO-CRC $\},\{$ PAY-SNR, ALG-YOU, FRB-MRC $\}$ and $\{$ PAY-SNR, ALG-YOU, MAB-SJM\}.

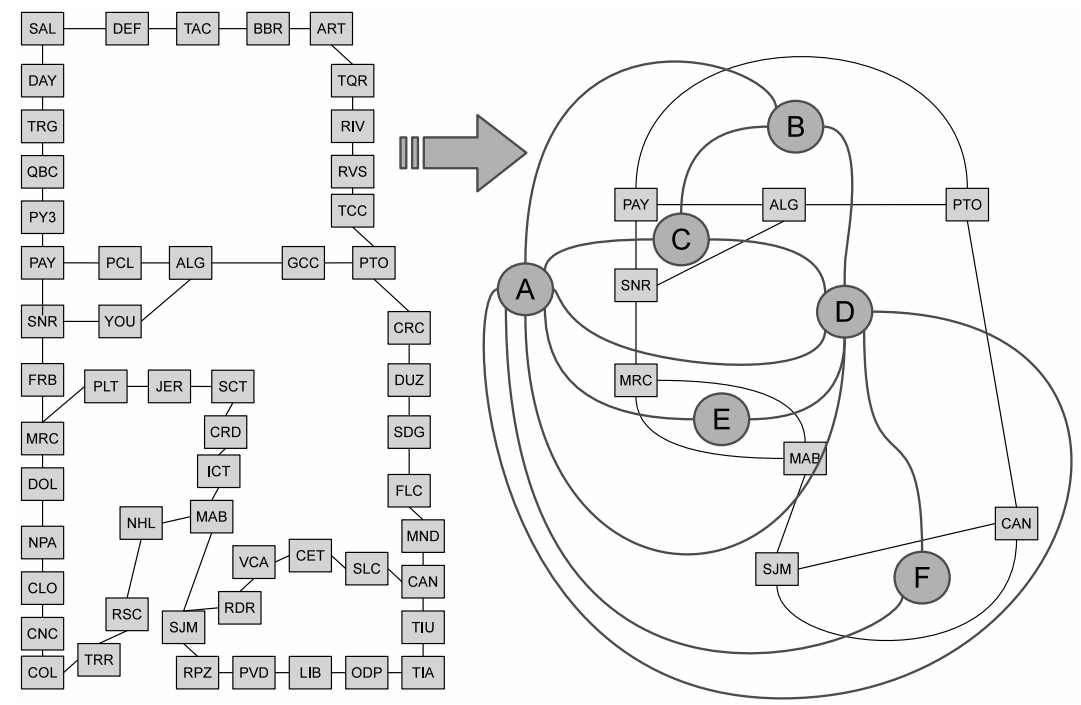

Figure 13 - Structural bones defined by dual cycles.

\subsubsection{Overlay routing}

This block aims to build a feasible configuration of lightpaths over the physical layer. Prior to do this a subset of input logical links must be chosen to be part of the solution. In this stage of the construction the emphasis lies on determining which inter-faces logical links are necessary. This process is very simple, for every structural bond (as seen in Section 5.3.2) a subset of the logical links that traverse this bond is randomly chosen in order to minimally satisfy equation (5).

The second step of this block, consists in performing a heuristic to construct a balanced-routing configuration of low-cost for the remaining logical links.

The goal is to find a path for every lightpath, such that the number of physical link intersections is minimum. It is also desirable that the total cost is as low as possible but as a second priority. Unfortunately, this subproblem is NP-Hard (see [8]) so a pseudo-aleatory heuristic was 
used. A detailed explanation of the heuristic implementation used to find the configuration of the lightpaths, can be seen at [15].

Regarding performance, there is an important feature of our implementation: it is much cheaper to discard a solution than accept it as a feasible one. This is because to validate a candidate solution, a different configuration must be found to route traffic in every failure scenario, whereas to discard it, we take the result of the heuristic that will be commented in Section 5.3.4. This heuristic usually stops very quickly when there is not enough capacity in some failure scenario. So, prior to pass to the local search phase, inter-faces links are reinserted selectively until regain feasibility.

\subsubsection{Local search}

In opposition to the strategy followed in the previous phase, in this one, we take each logical link of the solution - all of them, inter and intra-faces links - in decreasing order of cost - lightpaths costs - and attempt to remove it from the solution. If the result remains feasible, this link will be excluded from the solution; otherwise it will be reinserted. The process is repeated until all links are analyzed.

Although simple to express, the previous strategy lacks in performance if it is thoroughly applied. This is because the problem of finding an exact answer to the question: Is a given network able to route traffic under constraints of Section 3, is NP-Complete. Arguments regarding this fact were commented in the first part of Section 4.

Instead of an exact algorithm, our implementation used an efficient heuristic. Moreover, our heuristic is based on an improvement of the algorithm that commercial routers implement over IP/MPLS networks, for automatic routing with traffic engineering based on constraints. The basic algorithm is called Constrained Shortest Path First (CSPF), and our implementation improved the construction by sorting paths in decreasing order of traffic demand before routing.

This approximation integrates a well known heuristic used to solve NPP (Number Partition Problem), which is an NP-Complete problem from which we proved that the mere optimal routing over the logical layer was NP-Hard. For further details on CSPF refer to [20] and for NPP refer to [5].

Although it is possible for this heuristic to discard feasible solutions, it is unlikely. Further details of the heuristic implementation is out of the scope of this paper, but it can be accessed at [15].

\section{CONCLUSIONS}

The problem of designing an optimal and resilient IP/MPLS network over an existing optical infrastructure is very hard. It integrates and combines several NP-Hard problems into one. During the design and construction of algorithms we hit hard problems in most subroutines. Some of them are described in the results of Section 4, others, remarked during description of the GRASP algorithm (Section 5.3.4 and Section 5.3.3). Even the construction of the input data-set should 
be done carefully. Because of the complexity of this problem, it is not expectable to find good quality solutions manually.

Without opening reserved details, we must comment that results for scenarios summarized in Table 1, helped ANTEL in several ways. First of all, there were cases where savings coming from computed solutions exceeded 30\%. Moreover, manually designed networks suffered effects from physical faults more often and critically than solutions created using this metaheuristic. So the usage of this algorithm helped not only to save cost but also to improve the quality of the solutions.

For some aspects of design (questions of Section 5.1), it would have been impossible to have accurate answers without computer assistance. For example: allowing some changes to the current architecture, like merging both IP/MPLS networks into one, reported extraordinary improvements in the quality of the solutions. An extended analysis allowed us to isolate particular issues of the physical network responsible of these gaps. Many of these issues were fixed or are under process of correction.

We are confident that the context this work deals with is not an exception, and improvements should replicate from one ISP to the other. We are also confident that there is room to improve the quality of algorithms, by combining some of them into a hybrid. This is planned as future work.

Finally, we successfully integrated theoretical results during the design and implementation of some metaheuristics. GRASP was especially suitable to this, and we are confident that the outstanding results obtained with it, are based in the high level of tuning this metaheuristic allowed to introduce.

\section{ACKNOWLEDGMENTS}

The work of C. Risso, E. Canale and F. Robledo was partly supported by ANII and PEDECIBA, Uruguay. We also would like to thank to ANTEL (Administración Nacional de Telecomunicaciones from Uruguay) for its financial support to our work; and especially to: Diego Valle Lisboa and Laura Saldanha for helping us to define the models and gathering all the information necessary to feed and benchmark these algorithms.

\section{REFERENCES}

[1] Corez A \& Robledo F. 2011. Multi-overlay network planning by applying a Variable Neighborhood Search approach. Proceedings of IEEE 3rd Latin-American Conference on Communications 2011 (IEEE LATINCOM 2011), Belém do Pará, Brazil. p. 1-6.

[2] Eswaran KP \& TARJAn RE. 1976. Augmentation problems. Society for Industrial and Applied Mathematics (SIAM). Lecture Notes in Mathematics, 5(4): 653-665.

[3] Fioruci JA, Toledo FMB \& NASCimento MCV. 2012. Heuristics for minimizing the maximum within-clusters distance. Pesquisa Operacional, Dec 2012, 32(4): 497-522. Brazilian Operations Research Society. ISSN 0101-7438. 
[4] GAREY MR \& Johnson DS. 1979. Computers and Intractability: A Guide to the Theory of NPCompleteness. New York: W.H. Freeman. ISBN 0-7167-1045-5.

[5] Hayes B. 2002. The Easiest Hard Problem: Number Partitioning. American Scientist, 90(2): 113, March-April 2002, New York, USA.

[6] Kerivin H \& Mahjoub AR. 2003. Design of survivable networks: A survey. Networks, 46(1): $1-21$.

[7] Koster A, Orlowski S, RaAck C, Baier G \& Engel T. 2008. Single-layer Cuts for Multi-layer Network Design Problems. Springer-Verlag: Selected proceedings of the 9th INFORMS Telecommunications Conference, 44(1): 1-23.

[8] Oellrich M. 2008. Minimum Cost Disjoint Paths under Arc Dependence. Algorithms for Practice. PhD thesis: University of Technology, Berlin, Germany.

[9] OKamura H \& Seymour PD. 1981. Multicommodity flows in planar graphs. Journal of Combinatorial Theory, Series B, 31(1): 75-81.

[10] Orlowski S, Koster A, RAACK C \& Wess ÄLY R. 2007. Two-layer network design by branchand-cut featuring MIP-based heuristics. Proceedings of the 3rd International Network Optimization Conference (INOC 2007), Spa, Belgium.

[11] Pardalos PM \& Resende MGC. 2006 Handbook of Optimization in Telecommunication Springer Science + Business Media, New York. February 2006, ISBN: 0-38-730662-5.

[12] Parodi C. 2011 Integer Optimization Applied to the Design of Robust Minimum Cost Multi-Layer Networks. Master Thesis. Universidad de la República, Uruguay. http: / / premat. fing . edu . uy/IngenieriaMatematica/archivos/tesis_cecilia_parodi.pdf.

[13] RESENDE M \& Riberio C. 2003. Greedy randomized adaptive search procedures. ATT Research. http: / /www2 .research.att.com/ mgcr/doc/sgrasp-hmetah.pdf.

[14] Risso C, Nesmachnow S \& Robledo F. 2012. A Parallel Evolutionary Algorithm for Multilayered Robust Network Design. Selected proceedings of the 1st International Workshop on Soft Computing Techniques in Cluster and Grid Computing Systems (SCCG 2012), Victoria, Canada. p. 291-296.

[15] Risso C \& Robledo F. 2013. Using GRASP for designing a layer network. International Journal of Metaheuristics, Special Issue on Metaheuristics for Security, Reliability and Trust (IJMHEUR 2013). To appear.

[16] Robledo F. 2005. GRASP heuristics for Wide Area Network design. PhD thesis, Université de Rennes 1, 2005.

[17] Silva GC DA, OChI LS \& MARTINS SL. 2006. Proposta e avaliação de heurísticas GRASP para o problema da diversidade máxima. Pesquisa Operacional, Ago 2006, 26(2): 321-360. Brazilian Operations Research Society. ISSN 0101-7438.

[18] Stoer M. 1992. Design of survivable networks. Lecture Notes in Mathematics.

[19] WeSt DB. 1995. Introduction to Graph Theory. Theorem 7.1.12. Prentice Hall Professional Technical Reference, ISBN-13: 9780132278287.

[20] Ziegelmann M. 2007. Constrained Shortest Path and Related Problems. Constrained Network Optimization. VDM Verlag Dr. Müller. ISBN 978-3-8364-4633-4. 